\title{
Ankle Fractures: The Operative Outcome
}

\author{
Ahmad Hafiz Z, MS Orth, Nazri MY, MMed Orth, Azril MA, MMed Orth, Kassim NA, MBBS, \\ Nordin N, MBBS, Daraup S, MBBS, N Premchandran*, MS Orth \\ Department of Orthopaedics, Traumatology \& Rehabilitation, \\ International Islamic University Malaysia, Kuantan, Malaysia \\ *Department of Orthopaedic, Hospital Tengku Ampuan Afzan, Kuantan, Malaysia
}

\begin{abstract}
Ankle fractures are commonly seen in orthopaedic practice. This retrospective study of patients with ankle fractures who underwent surgical treatment in our institution from January 2000 to December 2003 was undertaken to analyze the common causes and patterns of ankle fractures; and the functional outcome of operative treatment for these fractures. Eighty patients were identified and reviewed. There were 65 male (81.3\%) and 15 female patients (18.7\%) with age ranging from 13 to 71 years old (mean, 32.3y). Common causes of ankle fractures were trauma (especially motor vehicle accidents), sports injuries and the osteoporotic bones in the elderly. Weber C (64.0\%) was the most common pattern of fracture at presentation. The most common operative treatment for ankle fractures was open reduction and internal fixation (73 patients, 91.2\%). Excellent and good outcomes were achieved in $93.8 \%$ of cases when measured using the Olerud and Molander scoring system for foot and ankle. In conclusion, operative treatment for ankle fractures restores sufficient stability and allowed mobility of the ankle joint.
\end{abstract}

Key Words:

Ankle Fracture, Operative Treatment, Functional Outcome

\section{INTRODUCTION}

Ankle fractures are commonly seen injuries in both the general population and in athletes. Common causes of ankle fracture are trauma (especially motor vehicle accidents). sports injuries (i.e., football players), and osteoporosis in the elderly (including postmenopausal women). Management of these fractures depends on the stability of the injury. Ankle fractures are often unstable. A stable fracture usually needs minimal splinting whereas unstable fractures may need open reduction and internal fixation. The objectives of this study were to analyze the causes and the patterns of ankle fractures as well as functional outcomes of surgically treated ankle fractures performed at our hospital from 2000 to 2003.

\section{MATERIALS AND METHODS}

This was a retrospective study of patients with ankle fractures who underwent surgical treatment in our hospital from January 2000 to December 2003; data was compiled using a combination of case note review, radiographic review and clinical assessment. Inclusion criteria were patients who had undergone surgical treatment and had complete case notes available for review, including plain radiographs, as well as continuous follow up at our hospital. Exclusion criteria were all patients with ankle fracture, who were treated conservatively, patients that were discharged for follow-up to other hospitals, and patients with pilon fractures. Data collected include age, length of hospital stay, use of antibiotics, pattern and site of the fractures, type of fixation, complications, and outcomes. Outcome measurements were determined using the Olerud and Molander scoring system ${ }^{1}$.

\section{RESULTS}

There were 208 cases of surgically treated ankle fractures at our hospital from January 2000 to December 2003. Of these, only 98 cases fulfilled the inclusion criteria. and 80 (78.4\%) patients were available for evaluation. Our hospital is a referral centre for the state of Pahang and the southern part of the state of Terengganu; thus more than half of the patients (110 patients from a total of 208) presented for postoperative follow up at the referring hospital upon discharge from the ward. The remaining 18 patients those were not included in the study because their case notes were unavailable due to pending court cases and insurance claims.

There were 65 male (81.3\%) and 15(18.7\%) female patients with an age range from 13 to 71 years old (mean age, 32.3y). Follow up ranged from 12 to 116 weeks ( 30.8 weeks). In most cases, patients stayed in the hospital for less than 2 weeks $(74.4 \%)$. A minority were hospitalized for more than 3 weeks. Some of these were polytrauma cases, and had complications such as infections. Some patients were having financial problems and were unable to pay for the implants, causing a delay in surgical treatment. 
Table I: Scoring system devised by Olerud and Molendar (maximum 100 points)

\begin{tabular}{|llr|}
\hline PARAMETER & DEGREE & SCORE \\
\hline 1. Pain & None & 25 \\
& While walking on uneven surface & 20 \\
& While walking on even surface outdoors & 10 \\
& While walking indoors Constant and severe & 5 \\
2. Stiffness & None & 0 \\
3. Swelling & Stiffness & 10 \\
& None & 0 \\
4. Stair-climbing & Only in evenings & 10 \\
& Constant & 5 \\
5. Running & No problems & 0 \\
6. Jumping & Impaired & 10 \\
7. Squatting & Impossible & 5 \\
& Possible & 0 \\
8. Supports & Impossible & 5 \\
& Possible & 0 \\
9. Work, activities of daily life & Impossible & 5 \\
& No problems & 0 \\
& Impossible & 5 \\
& None & 0 \\
& Taping, Wrapping & 10 \\
& Stick or crutch & 5 \\
\end{tabular}

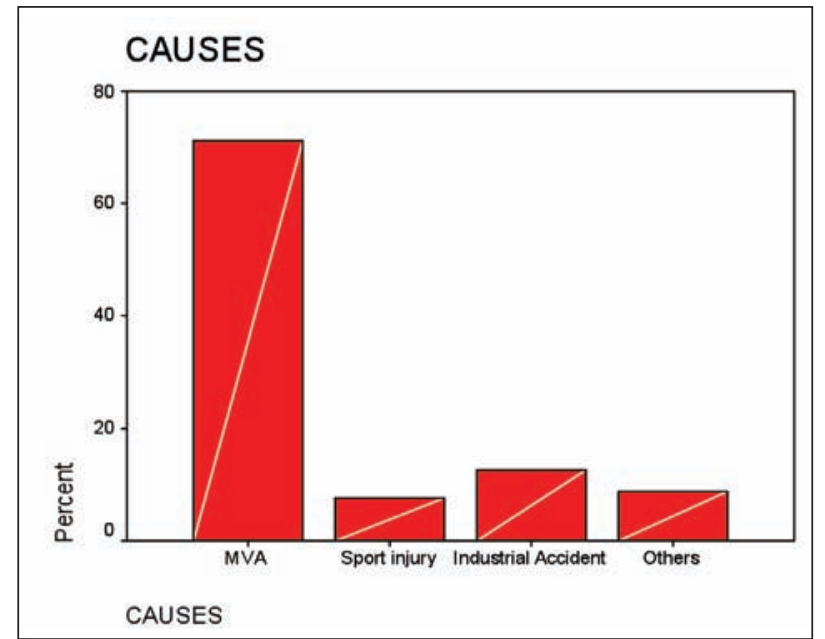

Fig. 1: The causes lead to ankle fractures.
Antibiotics are typically administered prophylactically surgery. In the present study, the most common antibiotics were intravenous cefuroxime $(41.1 \%$, a second generation cephalosporin); intravenous ceftriaxone $(32.9 \%$ a third generation cephalosporin); combinations including ceftriaxone or cefuroxime in combination with other antibiotics such as metronidazole $(26.0 \%)$.

\section{Causes}

Motor vehicle accident (MVA) was the most common cause of ankle fractures (70.4\% of all study subjects) (Figure 1). In young, active people, fractures were associated with vigorous activity, especially sports $(6.2 \%)$. Other causes include falls, especially in elderly $(8.6 \%)$. Among the MVA cases $45.7 \%$ were motorcyclists and $1.2 \%$ were lorry drivers.

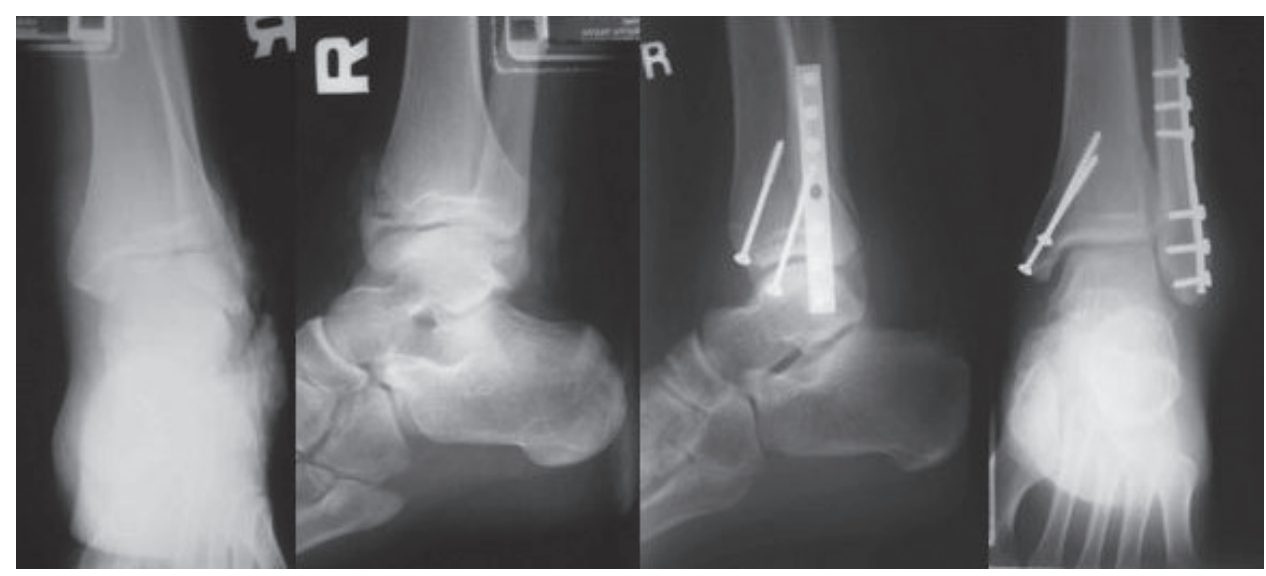

Fig. 2: Preoperative and post-operative plain radiograph. 


\section{Pattern and Site of ankle fractures}

Almost two-third of all ankle fractures cases were closed fractures (61 cases, 76.3\%) and there were 19 cases of open ankle fractures (23.7\%). The right side (58.8\%) was affected slightly more than the left $(41.2 \%)$. The medial malleoli $(42.5 \%)$ was the most commonly injured bone of the ankle and the least common was the posterior malleoli $(2.5 \%)$. Based on the Dennis- Weber classification, type-C was the most common $(64.0 \%)$ fracture pattern that required operative treatment.

\section{Types of fixation}

The most common operative method was open reduction and internal fixation (ORIF) in 73 patients $(91.2 \%)$ with open reduction followed by external fixation in only 7 patients $(8.8 \%)$. ORIF is the treatment of choice for patients with displaced fractures of both the lateral and medial malleolus. The most common implants used in medial malleolus fractures were screws (71.6\%), whereas for lateral malleolus fractures, plates were the common choice $(56.1 \%)$. There were no implants used for the posterior malleolus fractures in our study. (Figure 2)

\section{Complications}

The most common complications were superficial infection (12 cases, 15\%); deep infection leading to non-union (2 cases, $2.5 \%$ ) followed by joint stiffness ( 1 case, $1.2 \%$ ). All superficial and deep infections occurred in cases of open fractures. All superficial infection resolved with local dressing and systemic antibiotics. Deep infections that occurred resulted in poor outcomes - cases with open grade 111 A were badly contaminated and had continuing problems with stiffness.

\section{Patient Outcomes}

Using the Olerud and Molander scoring system (maximum 100 points), we note that $93.8 \%$ of patients had excellent and good outcomes (Table 1) ${ }^{1}$. There were 3 patients $(3.7 \%)$ with poor results, $2(2.5 \%)$ fair, one good $(1.3 \%)$ and 74 (92.4\%) excellent results. Two cases of poor results were due to deep infection, and the other case was a 71 years old with stiffness and ankle pain when walking

\section{DISCUSSION}

Operative treatment for ankle fractures restores sufficient stability to allow full mobility at the ankle joint. Makwana et al compared 22 cases of ORIF and 21 cases of conservatively treated patients with ankle fractures and found that ORIF treatment yielded a significantly higher functional outcome score and a significantly better range of movement of the ankle ${ }^{2}$. The Ponzer et al study demonstrated satisfactory outcomes in $76 \%-83 \%$ of operatively treated type-B malleolar injuries ${ }^{3}$. Although we did not compare the outcome of patients treated conservatively vs. operatively, we were able to achieve excellent and good results in $93.8 \%$ (75 cases) of the cases. We had a much younger age group in the present study compared to the other studies. The Makwana et al study included ankle patients over 55years in age ${ }^{2}$. Our study showed Weber type-C (64.0\%) was the most common presentation.

Some studies reported that infections were common after foot and ankle surgery, however Miller et $a l^{4}$ reported infections in $2.2 \%$ of 1841 patients after foot and ankle surgery And Hugar et $a l^{5}$ reported infections in $1.35 \%$ of patients after outpatient foot surgery ${ }^{4,5}$. We found a slightly higher rate of infection in the present study $(3.7 \%)$. Antibiotic coverage is usually based on the severity of the soft tissue injury and the grade of the open fracture ${ }^{6}$. The risk of infection after internal fixation of open or closed fractures can be decreased by the use of antimicrobial prophylaxis and proper surgical technique that includes proper soft tissue handling during placement of the implant ${ }^{7}$. Infection following foot and ankle surgery or trauma can range from commonly occurring superficial cellulitis to less common deep soft tissue or bone infections that can have disastrous consequences. Infection is the most important factor in the development of non-union, loss of function and other complications after foot and ankle trauma and prevention of infection is essential to obtaining bony union and soft tissue coverage that results in a functional plantigrade foot ${ }^{8}$.

\section{CONCLUSIONS}

Operative treatment for ankle fractures results good functional outcome post operatively, and operative treatment restores sufficient stability and allowed good mobility of the ankle joint. Specific measures must be taken to reduce the number of superficial or deep infections. Other modes of treatment may be considered in cases with severe contamination. Motorcyclists must be aware of risks of ankle fracture and safety measures should be taken to prevent ankle fractures among motorcyclists. 


\section{REFERENCES}

1. Olerud C, Molander H. A Scoring scale for symptom evaluation after ankle fracture. Arch Orthop Trauma Surg 1984; 103 : $190-4$.

2. Makwana N, Bhowal B, Harper WM, Hui AW. Conservative versus operative treatment for displaced ankle fractures in patient over 55 years of age: a prospective randomized study. J Bone Joint Surg 2001; 83B: 525-9.

3. Ponzer S, Nsell H, Bergman B, Trnkvist H. Functional Outcome and Quality of Life in Patients with Type B Ankle Fractures: A Two-Year Follow-up Study. J OrthopTrauma 1999; 13(5): 363-8.

4. Miller WA. Postoperative wound Infection in Foot and Ankle Surgery. Foot Ankle 1983; 4: 102-4.

5. Hugar DW, Newman PS, Hugar RW, Spencer RB, Salvino K. Incidence of Postoperative Infection in a Free-standing ambulatory Surgery Centre. J Foot Surg 1990; 29: 265-7.

6. Flynn JM, Rodriguez-del Rio F, Piza PA. Closed Ankle Fractures in the Diabetic Patient, Foot Ankle Int 2000; 21 : 311-9.

7. Worlock P, Slack R, Harvey L, Mawhinney R. The Prevention of Infection in Open Fractures: An Experimental Study of the Effect of Fracture Stability. Injury 1994; 25: 31-8.

8. Donley BG, Philbin T, Tomford JW, Sferra JJ. Foot and Ankle Infections after Surgery. Clin Orthop Relat Res 2001; 391: 162-70.

9. Fehily M, Fleming P, Yousef M, Khan R, Mohoklar K, Borton D. Weight-bearing Following Ankle Fractures- A Study of Patient Compliance. J Bone Joint Surg 2002; 84B: 4-5. 